\title{
Tire Road Friction Coefficient Estimation: Review and Research Perspectives
}

\author{
Yan Wang ${ }^{1}$, Jingyu Hu' ${ }^{1}$, Fa'an Wang ${ }^{1}$, Haoxuan Dong ${ }^{1}$, Yongjun Yan ${ }^{1}$, Yanjun Ren ${ }^{1}$, Chaobin Zhou ${ }^{1,2}$ and \\ Guodong Yin ${ }^{1 *}$ (D)
}

\begin{abstract}
Many surveys on vehicle traffic safety have shown that the tire road friction coefficient (TRFC) is correlated with the probability of an accident. The probability of road accidents increases sharply on slippery road surfaces. Therefore, accurate knowledge of TRFC contributes to the optimization of driver maneuvers for further improving the safety of intelligent vehicles. A large number of researchers have employed different tools and proposed different algorithms to obtain TRFC. This work investigates these different methods that have been widely utilized to estimate TRFC. These methods are divided into three main categories: off-board sensors-based, vehicle dynamics-based, and data-drivenbased methods. This review provides a comparative analysis of these methods and describes their strengths and weaknesses. Moreover, some future research directions regarding TRFC estimation are presented.
\end{abstract}

Keywords: Intelligent vehicles, Tire road friction coefficient (TRFC), Off-board sensors-based method, Vehicle dynamics-based method, Data-driven-based method

\section{Introduction}

Traffic accidents are one of the leading causes of injuries and death in China and abroad. According to the National Bureau of Statistics of China, in 2019, there were 247646 traffic accidents, which resulted in 62763 fatalities, 256101 injuries, and a direct economic loss of 1346.18 million yuan. Therefore, both industry and academia have made great efforts to develop new technologies to reduce or even avoid traffic accidents. Active safety systems are the most representative of these new technologies, which include antilock braking systems [1], electronic stability control systems [2, 3], active collision avoidance systems [4], etc.

The main function of antilock braking systems is to prevent the wheel lock during heavy braking and to maintain the traction between the tires and the road at an optimal value. The magnitude of this optimal traction

\footnotetext{
*Correspondence: ygd@seu.edu.cn

${ }^{1}$ School of Mechanical Engineering, Southeast University,

Nanjing 211189, China

Full list of author information is available at the end of the article
}

is usually determined based on the tire road friction coefficient (TRFC). The electronic stability control systems generate a yaw moment based on the desired yaw rate to ensure the lateral stability of the vehicle. The desired yaw rate normally shows a positive correlation with TRFC. The active collision avoidance systems use a variety of sensors to obtain information about the surrounding environment of the vehicle to reduce the risk of accidents. Active collision avoidance systems will work when the relative distance between the vehicle and the obstacle is lower than the safety distance. This safety distance is negatively correlated with TRFC. The above analysis shows that accurate TRFC information is essential to improve the performance of active safety systems. Unfortunately, TRFC cannot be measured by on-board sensors. To this end, researchers have successively proposed various approaches to address the challenge. Refs. [5-7] also provide a review of the models and methods used for TRFC estimation. However, few types of research have systematically discussed the acquisition of TRFC from the perspective of off-board sensors-based, vehicle dynamics-based, data- driven-based. 
This article systematically reviews the recent developments on TRFC estimation from different research directions. It contains a comparative analysis of existing methods and describes their strengths and weaknesses. Also, some future research directions regarding TRFC estimation are presented. Based on this, we believe this work will help the researcher or vehicle engineer to choose a suitable approach for TRFC estimation.

To give some details of the analysis, the rest of this article is organized as follows. In Section 2, the different types of TRFC estimation methods are presented. In addition, the advantages and shortcomings of these existing methods are briefly elaborated. The conclusion and some promising prospects regarding TRFC estimation are given in Section 3.

\section{TRFC Estimation Methods}

There are three main directions of existing research on TRFC estimation including identification methods based on off-board sensors, vehicle dynamics-based approaches, and data-driven prediction methods. These estimation methods are divided into three groups according to different categories as shown in Figure 1.

\subsection{Off-board Sensors-based Methods}

Studies have shown that changes in micro/macro-texture of road surfaces affect TRFC [8], which has prompted many scholars to use this knowledge to obtain TRFC. Estimation methods based on this principle usually require the use of a camera to acquire a certain amount of road images and subsequently utilize some algorithms to obtain the TRFC. Baffet et al. [9] used multiple linear regression analysis and the fuzzy logic method to estimate the TRFC. Du et al. [10] proposed a deep neural network based on domain knowledge for estimating TRFC. Leng et al. [11] developed a fusion strategy of a dynamic estimator and visual estimator to identify TRFC. Yu et al. [12] made use of a backpropagation (BP) neural network to predict TRFC. This method usually has better estimation accuracy in high visibility environments while the prediction performance decreases significantly in night driving environments.

To make the estimation algorithm work in a night driving environment, several estimation methods based on the physical deformation of the tire have been proposed successively. Some scholars found that tire deformation and vibration are also related to TRFC. Some sensors such as accelerometers are installed inside the tires (see Figure 2) to measure some key information to obtain TRFC.

Singh et al. [14] proposed a method to predict TRFC using the frequency response of tire vibration. A method of using acceleration information from intelligent tires

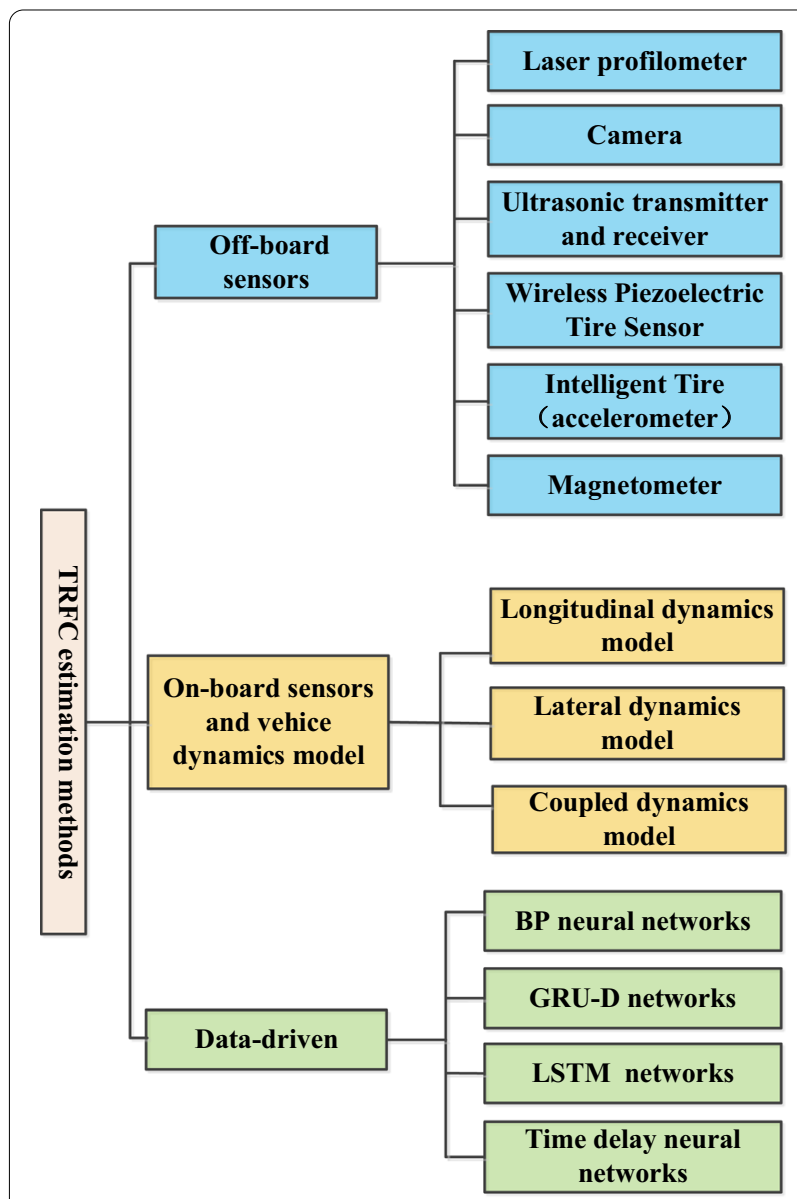

Figure 1 Classification of the TRFC estimation methods

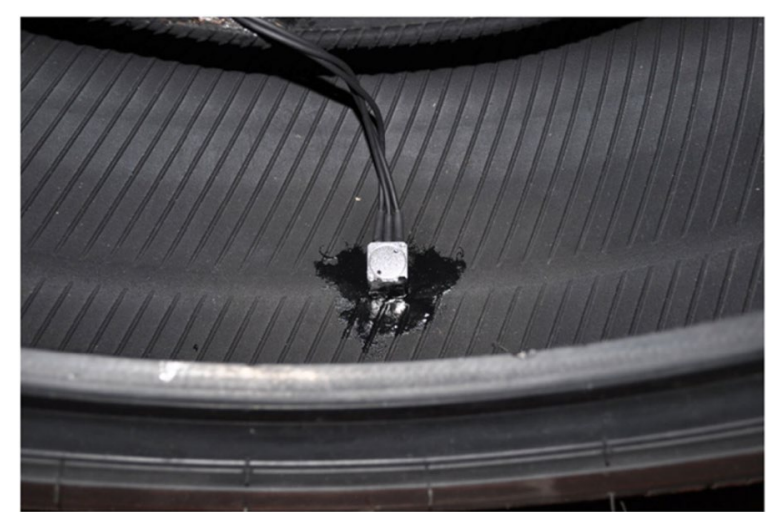

Figure 2 Using accelerometers to identify TRFC [13]

was presented in Refs. [13, 15, 16], and experimental results proved the effectiveness of this approach. However, due to the complex working conditions of tires, the sensors inside the tires are easily dislodged or damaged, which limits the further practical application of 
such methods. Furthermore, some estimation methods based on ultrasonic sensors[17], laser profilometer [18], wireless piezoelectric tire sensor [19], and magnetometer [20] have also been reported. The advantages of these offboard sensor-based methods are that they require fewer measurement variables, are insensitive to the vehicle's dynamic response, and do not require specific excitation inputs. Obviously, these off-board sensors need to be additionally assembled on series production cars. Also, the off-board sensor-based method can only obtain the approximate range of the TRFC and the measurement accuracy is sensitive to the interference of the external environment. For these reasons, vehicle dynamics-based methods have received increasing attention in recent years.

\subsection{Vehicle Dynamics-based Approaches}

The vehicle dynamics-based method identifies TRFC according to the dynamic response of the vehicle on different road surfaces. The vehicle dynamics-based approaches can be divided into three categories: longitudinal dynamics-based, lateral dynamics-based, and coupled dynamics-based methods.

\subsubsection{Longitudinal Dynamics-based Methods}

Longitudinal dynamics-based estimation methods typically have high estimation accuracy for acceleration and braking conditions. The principle underlying most of these estimation methods is the relationship between longitudinal slip and TRFC.

Figure 3 shows the relationship between the TRFC and the longitudinal slip for a variety of road surfaces. From Figure 3, we can see that TRFC is an increasing function of slip. As the slip increases, TRFC will reach a maximum value and then decrease slightly. Gustafsson et al. [21] first proposed a classical approach to estimate TRFC utilizing the longitudinal slip slope. In Refs. [21, 22], an

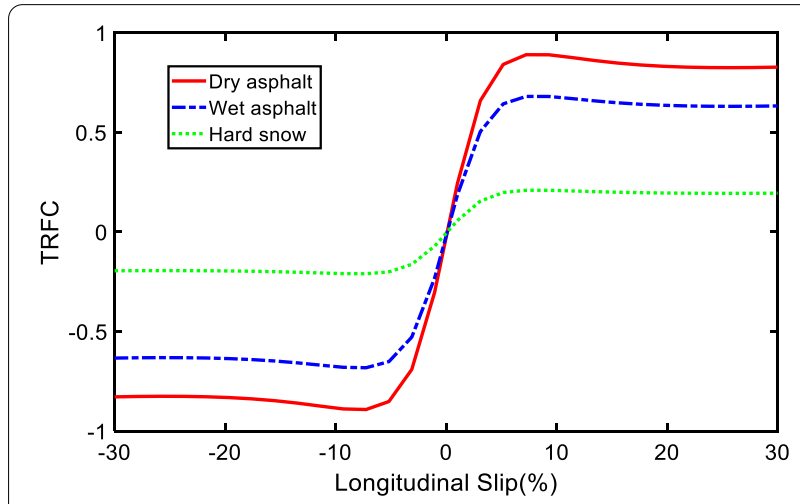

Figure 3 Longitudinal slip versus TRFC adaptive filter based on Kalman filter theory by using a magic formula (MF) tire model is proposed to estimate TRFC. Similarly, Yi et al. [23, 24] supplied more experimental data to sustain Gustafsson's view. Based on Gustafsson's idea, Rajamani et al. $[25,26]$ further extended the applicability of the method by adding global positioning system (GPS) signals. In addition, a rule-based TRFC estimation method was also proposed in Ref. [27], which can still have a good estimation accuracy when the wheels reach the limit of adhesion. Since TRFC varies not only with road conditions but also with tires, the experiment-based real-time TRFC estimation method can further improve the accuracy of TRFC estimation under different driving conditions [28]. Although the slip-based TRFC estimation method requires few sensors and shows promising results, it has major problems in terms of robustness and calibration. Accordingly, nonlinear curve fitting techniques were presented to address this problem [29-33]. In addition, another mainstream estimation method is to determine the TRFC from the obtained tire force. Some multiple analytical models were used to identify the TRFC in Refs. [34-40]. State observers and recursive least squares (RLS) are also used for TRFC estimation. For example, state observers based on the LuGre dynamic model [41-43], the Burckhardt model [44-47], the Magic Formula model [48, 49], the quarter wheel model [50], and the tire torsion model [51] were designed to identify the TRFC. RLS methods, which are mainly based on the brush model [37], the Burckhardt model [52, 53], longitudinal vehicle model [54-58], single-wheel model [59] and six-degree-of-freedom (DOF) vehicle model [60], have been extensively studied in recent years. It is well known that the RLS usually has only one degree of freedom to adjust the adaptivity of the filter, which may limit its application.

To fill the gap, Kalman filter-based methods are attracting more and more attention. Krisztian et al. [61] made use of extended Kalman filter for TRFC estimation. Castillo et al. [62] developed a TRFC estimation method incorporating fuzzy logic and a Kalman filter. In addition to the common methods mentioned above, some interesting estimation methods have been proposed by scholars. TRFC prediction method based on tire force information using conditional probability theory was presented in Ref. [63]. Resonance frequencybased TRFC estimation methods were developed in Refs. [64, 65]. A fuzzy logic-based TRFC identification method was presented in Ref. [66]. Also, the transformation of the TRFC estimation problem into an optimization problem is an interesting research direction [67]. To compare the various methods more clearly, the estimation methods based on vehicle longitudinal dynamics are presented in Table 1. 
Table 1 Summary of estimation methods based on vehicle longitudinal dynamics

\begin{tabular}{|c|c|c|c|}
\hline Number & Models & Methodology & References \\
\hline 1 & Torque-to-force transfer function & Analytical model & {$[34-36,39,40]$} \\
\hline 2 & Anisotropic brush model & Least square method & [37] \\
\hline 3 & Magic formula model & Kalman filter & {$[21,22]$} \\
\hline 4 & Eight DOF vehicle model & Extended Kalman-Bucy filtering & {$[63]$} \\
\hline 5 & Magic formula model & Reduced order observer & {$[23,24]$} \\
\hline 6 & Magic formula model & Analytical model & {$[38]$} \\
\hline 7 & Torque-to-force transfer function & State observer & {$[28]$} \\
\hline 8 & LuGre dynamic model & State observer & {$[41,42]$} \\
\hline 9 & Magic formula model & RLS & {$[25,26]$} \\
\hline 10 & Magic formula model & Nonlinear curve fitting technique & [29] \\
\hline 11 & Pseudostatic lugre model & Sliding mode observer & [43] \\
\hline 12 & Burckhardt model & Recursive least squares & [52] \\
\hline 13 & Tire torsion model & Sliding mode observer & [51] \\
\hline 14 & Longitudinal vehicle model & Nonlinear curve fitting technique & {$[30,32,33]$} \\
\hline 15 & Burckhardt model & Nonlinear curve fitting technique & {$[44,45]$} \\
\hline 16 & Longitudinal vehicle model & RLS & {$[54,56-58]$} \\
\hline 17 & Single wheel model & RLS & [59] \\
\hline 18 & Magic formula model & Improved nonlinear observer & [48] \\
\hline 19 & Burckhardt model & Sliding mode observer & {$[46]$} \\
\hline 20 & Six DOF vehicle model & RLS & {$[60]$} \\
\hline 21 & Three DOF vehicle model & Extended Kalman filter & [61] \\
\hline 22 & Longitudinal vehicle model & Proportional integral observer & {$[55]$} \\
\hline 23 & Quarter wheel model & Nonlinear Lipschitz observer & {$[50]$} \\
\hline 24 & Four wheel vehicle model & Fuzzy logic and Kalman filter & {$[62]$} \\
\hline 25 & Frequency response function & Analytical model & {$[64,65]$} \\
\hline 26 & Modified Burckhardt tire model & Nonlinear estimator & [47] \\
\hline 27 & Magic formula model & Genetic algorithm & {$[67]$} \\
\hline 28 & Novel TRFC slip curve & Nonlinear curve fitting technique & [31] \\
\hline 29 & Longitudinal vehicle model & Fuzzy logic & {$[66]$} \\
\hline 30 & Magic formula model & Linear extended state observer & [49] \\
\hline 31 & Longitudinal vehicle model & Rules-based method & [27] \\
\hline 32 & Lugre and Burckhardt models & T-S fuzzy and RLS & [53] \\
\hline
\end{tabular}

\subsubsection{Lateral Dynamics-based Methods}

Estimation approaches that consider vehicle longitudinal dynamics commonly require larger excitation, while methods based on vehicle lateral dynamics may also yield better estimation results when the excitation is relatively small. The general framework of TRFC estimation based on vehicle lateral dynamics is shown in Figure 4. When the driver applies a steering maneuver to the vehicle, several key state variables are first measured by on-board sensors. Then, after obtaining measurement signals, the lateral force of the tire is estimated by some advanced algorithms. Finally, the tire model and tire lateral force are used to predict TRFC.

Based on this general framework, some interesting estimation algorithms have been proposed to estimate TRFC. An analytical model based on lateral acceleration

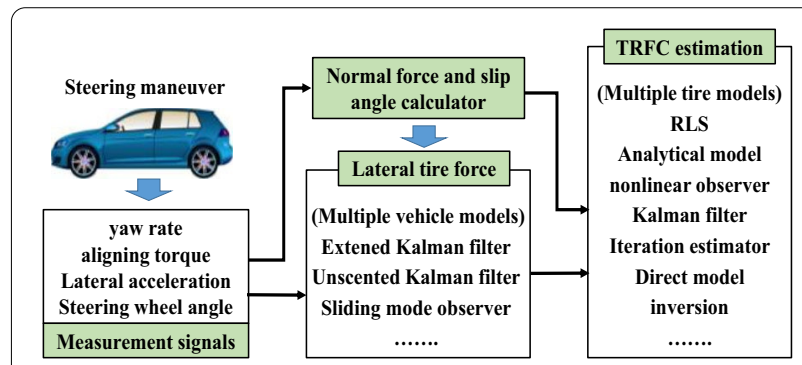

Figure 4 The general framework of TRFC estimation based on vehicle lateral dynamics.

to estimate TRFC was presented in Refs. [68, 69], and the method has good estimation results in the linear range of the tire. To further extend the scope of application of this 
method, an analytical model based on cornering stiffness coefficient to identify TRFC at large slip angles was developed in Ref. [70]. The use of yaw rate and aligning torque information to estimate TRFC was also presented in Refs. [71-73] respectively.

To reduce reliance on in-vehicle sensor information, Rajamani et al. [74] only used a differential global positioning system (DGPS) to identify TRFC. Erdogan et al. [75] utilized a new tire force measurement device to estimate TRFC without using information from the braking system. Although using less sensor information to estimate TRFC can help reduce costs in some normal driving conditions, the accuracy of TRFC estimation may be decreased in some complex driving situations. An integrated TRFC estimation strategy [76-78] was proposed using sensor fusion techniques to address this problem.

In addition, due to the coupling relationship between the vehicle state and TRFC, some scholars usually carry out the identification of sideslip angle and TRFC simultaneously to improve the estimation accuracy. An RLS algorithm for estimating the vehicle sideslip angle and TRFC was developed in Refs. [79, 80]. The Kalman filter as a special form of RLS has obvious advantages in dealing with estimation problems with measurement noise. Considering the sensor measurement noise interference and the nonlinearity of vehicle dynamics, an estimation method based on the double extended Kalman filter (EKF) was presented in Refs. [81, 82]. The unscented Kalman filter avoids solving the Jacobian matrix and can obtain higher estimation accuracy than EKF. Hence, the UKF was used for the estimation of TRFC [83]. However, these Kalman-based methods are only valid for Gaussian-distributed noise. For non-Gaussian and nonlinear systems, particle filters have high estimation accuracy. Liu et al. [84] proposed a prediction method combining auxiliary particle filter and iterative estimator and verified the effectiveness of the algorithm by real vehicle test.

On the other hand, methods using state observers are often reported such as extended Luenberger observer [85], online gradient descent algorithm [86], nonlinear observer [87, 88], high-order sliding mode observer [89], etc. The observer-based approach usually has a certain range of applicability, and an adaptive observer [90,91] for all road conditions was proposed to solve this problem. To compare the various methods more clearly, the estimation methods based on vehicle lateral dynamics are presented in Table 2.

\subsubsection{Coupled Dynamics-based Methods}

The above studies only considered vehicle longitudinal or lateral dynamics, which may result in a serious underestimation of the TRFC [92]. To increase the estimator working range, hybrid estimators began to be proposed one after another. The general framework of hybrid estimators is shown in Figure 5. This hybrid estimator improves the estimation accuracy by designing some hybrid algorithms to weigh the estimation results from different modules. Shim et al. [93] proposed a fusion method

Table 2 Summary of estimation methods based on vehicle lateral dynamics

\begin{tabular}{|c|c|c|c|}
\hline Number & Models & Methodology & References \\
\hline 1 & Single-track vehicle model & Analytical model & {$[71]$} \\
\hline 2 & Brush model & Analytical model & {$[68]$} \\
\hline 3 & Single-track vehicle model & Parameter identification algorithm & {$[74]$} \\
\hline 4 & Lateral tire forces & Analytical model & {$[75]$} \\
\hline 5 & Seven DOF vehicle model & Multi-sensor signal fusion method & {$[76-78]$} \\
\hline 6 & Single-track vehicle model & RLS & {$[79,80]$} \\
\hline 7 & Single-track vehicle model & Analytical model & {$[69]$} \\
\hline 8 & Brush model & Analytical model & {$[72]$} \\
\hline 9 & Three DOF vehicle model & Dual extended Kalman filter & {$[81,82]$} \\
\hline 10 & Nonlinear vehicle model & Switched multiple nonlinear observer & {$[90]$} \\
\hline 11 & Single-track vehicle model & Analytical model & {$[70]$} \\
\hline 12 & Random-walk model & Extended Luenberger observer & {$[85]$} \\
\hline 13 & Single-track vehicle model & Iteration estimator & {$[84]$} \\
\hline 14 & Seven DOF vehicle model & Online gradient descent algorithm & {$[86]$} \\
\hline 15 & Single-track vehicle model & Nonlinear observer & {$[87,88]$} \\
\hline 16 & Single-track vehicle model & Nonlinear adaptive observer & {$[91]$} \\
\hline 17 & Hypothetical brush model & Direct model inversion & {$[73]$} \\
\hline 18 & Single-track vehicle model & High-order sliding mode differentiator. & {$[89]$} \\
\hline 19 & Single-track vehicle model & Unscented Kalman filter & [83] \\
\hline
\end{tabular}




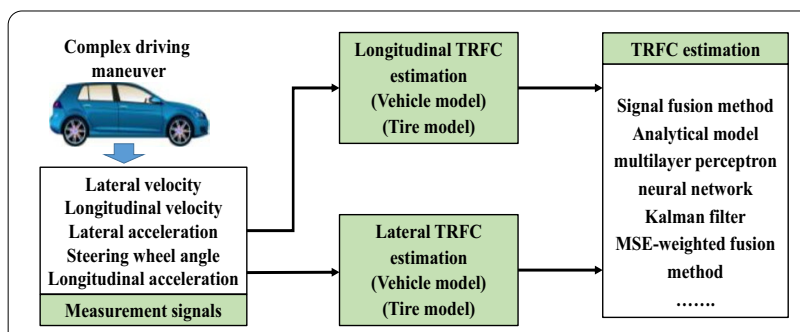

Figure 5 The general framework of the hybrid estimator

based on steering angle information to estimate TRFC. Villagra et al. [94] used new algebraic filtering techniques to consecutively estimate tire forces and TRFC. Ahn et al. [95] developed an integration logic to switch among the developed algorithms based on the nature and level of excitations. Ren et al. [96] designed an integrated estimator to predict TRFC on basis of information about longitudinal, lateral, and yaw motions.

To ensure that the estimator can adapt to complex and variable working conditions, a moving horizon estimation strategy [97] was proposed to estimate TRFC. In addition, time-domain-based signal fusion methods $[98,99]$ have also proven to be effective in dealing with the TRFC estimation problem. However, time-domainbased signal fusion methods usually have degraded estimation performance at small acceleration conditions. A frequency-domain data fusion was proposed to estimate the TRFC based on the natural frequencies of the steering system and the in-wheel motor driving system [100]. RLS-based methods were also proposed to dynamically predict TRFC based on longitudinal and lateral tire forces in Refs. [92, 101, 102]. Since Kalman filtering has a significant advantage over RLS in dealing with the estimation problem with measurement noise. Considering the nonlinearity of vehicle dynamics, an identification method based on the EKF was presented in Refs. [103, 104]. To reduce the influence of old measurement data on the filtering in the EKF algorithm, a limited-memory adaptive extended Kalman Filter [105] was proposed to solve the problem. Also, UKF [106] can obtain higher accuracy when dealing with nonlinear system state estimation, and it has also been used for TRFC estimation in recent years. Furthermore, due to the bad adaptability of traditional Kalman filters to variable system structure, an improved Strong Tracking UKF [107] was constructed to identify the TRFC. To reduce the workload required for mathematical derivations of the Kalman filtering method, a nonlinear state observer was proposed to estimate TRFC [108, 109]. In addition to some of the improved fusion strategies discussed above, neural network-based fusion methods [110] were also an interesting research direction. To compare the various methods more clearly, the estimation methods based on vehicle coupled dynamics are presented in Table 3 . The advantage of the vehicle dynamics-based method is that the TRFC can be estimated using on-board sensors; the estimation cost is low, and the real-time performance is effective. The estimated

Table 3 Summary of estimation methods based on vehicle coupled dynamics

\begin{tabular}{|c|c|c|c|}
\hline Number & Models & Methodology & References \\
\hline 1 & Four wheel vehicle model & Analytical model & {$[93]$} \\
\hline 2 & Four wheel vehicle model & Unscented Kalman filter & {$[106]$} \\
\hline 3 & Kinematic model & Analytical model & {$[94]$} \\
\hline 4 & Single-track vehicle model & Analytical model & {$[95]$} \\
\hline 5 & Brushed tire model & Linearized RLS & {$[92]$} \\
\hline 6 & Three DOF vehicle model & Extended Kalman filter & {$[103]$} \\
\hline 7 & Three DOF vehicle model & Integrated estimator & {$[96]$} \\
\hline 8 & Planar vehicle model & RLS & {$[102]$} \\
\hline 9 & Three DOF vehicle model & Signal fusion method & [98] \\
\hline 10 & Four-DOF vehicle model & Extended Kalman filter & {$[104]$} \\
\hline 11 & Planar vehicle model & MSE-weighted fusion method & [99] \\
\hline 12 & Three DOF vehicle model & RLS & {$[101]$} \\
\hline 13 & Fourteen DOF vehicle model & Multilayer perceptron neural network & {$[110]$} \\
\hline 14 & Active front steering model & Frequency domain data fusion & {$[100]$} \\
\hline 15 & Planar vehicle model & Nonlinear observer & {$[108]$} \\
\hline 16 & Three DOF vehicle model & Limited-memory adaptive EKF & {$[105]$} \\
\hline 17 & Kinematic model & Nonlinear observer & {$[109]$} \\
\hline 18 & Three DOF vehicle model & Moving horizon estimation strategy & {$[97]$} \\
\hline 19 & Seven DOF vehicle model & Improved strong tracking UKF & [107] \\
\hline
\end{tabular}


TRFC can meet the needs of advanced chassis control with guaranteed vehicle model accuracy. It is well known that many assumptions and corresponding mathematical simplifications need to be made before building a vehicle dynamics model. Some idealized assumptions will increase the inaccuracy of the vehicle model and thus affect the accuracy of TRFC estimation, especially for some extreme driving conditions.

\subsection{Data Driven-based Approaches}

To compensate for the shortcomings of the vehicle dynamics-based method, neural networks were used to describe the tire and wheel suspension behavior [111]. A genetic algorithm optimized neural network is then employed to identify the TRFC. A similar idea was also reported in Ref. [112]. Zhang et al. [113] developed a mapping from input parameters to TRFC using general regression neural network, which effectively avoids storing complex tire models. Ribeiro et al. [114] employed the time-delay neural network to estimate TRFC can avoid using the standard tire mathematical model which makes the estimation method more robust. In addition, other neural networks have also been applied to the estimation of TRFC such as gated recurrent unit (GRU) network [115], deep neural network [116], BP neural network [12], deep convolutional neural network [17], etc. Furthermore, the time series characteristics of TRFC are not considered in the above data-driven estimation method. A long-short term memory (LSTM) neural network [117] was developed to address this problem. In general, with the enhancement of the computing power of the central processing unit, data-driven methods have received more and more attention. However, it should be noted that the prediction accuracy of such methods relies on the completeness of the dataset, and a comprehensive dataset is usually more difficult to obtain in practice. In addition, the generalization capability of the data-driven approach may further affect its applicability.

\section{Summary and Perspectives}

In this article, we review and compare typical TRFC estimation approaches. Three types of TRFC estimation methods have been systematically assessed and summarized. Although many outcomes have been achieved in TRFC estimation, some interesting points should be noted for future research. The first and foremost idea is to combine the advantages of various methods to improve the estimation accuracy of TRFC. Secondly, with the advent of the Internet of Things era, intelligent connected vehicles are gradually moving from the laboratory to public roads. Vehicles can exchange information with various traffic elements to obtain the friction coefficient of surrounding roads. In addition, with the development of prediction theory, predicting road friction in the future period is becoming a reality. Based on the above discussion, future studies on TRFC estimation are shown in Figure 6.

The development of technology and theory allows us to use advanced sensors and algorithms to estimate TRFC and gradually improve the estimation accuracy from various aspects. Some future research directions on TRFC estimation are as follows.

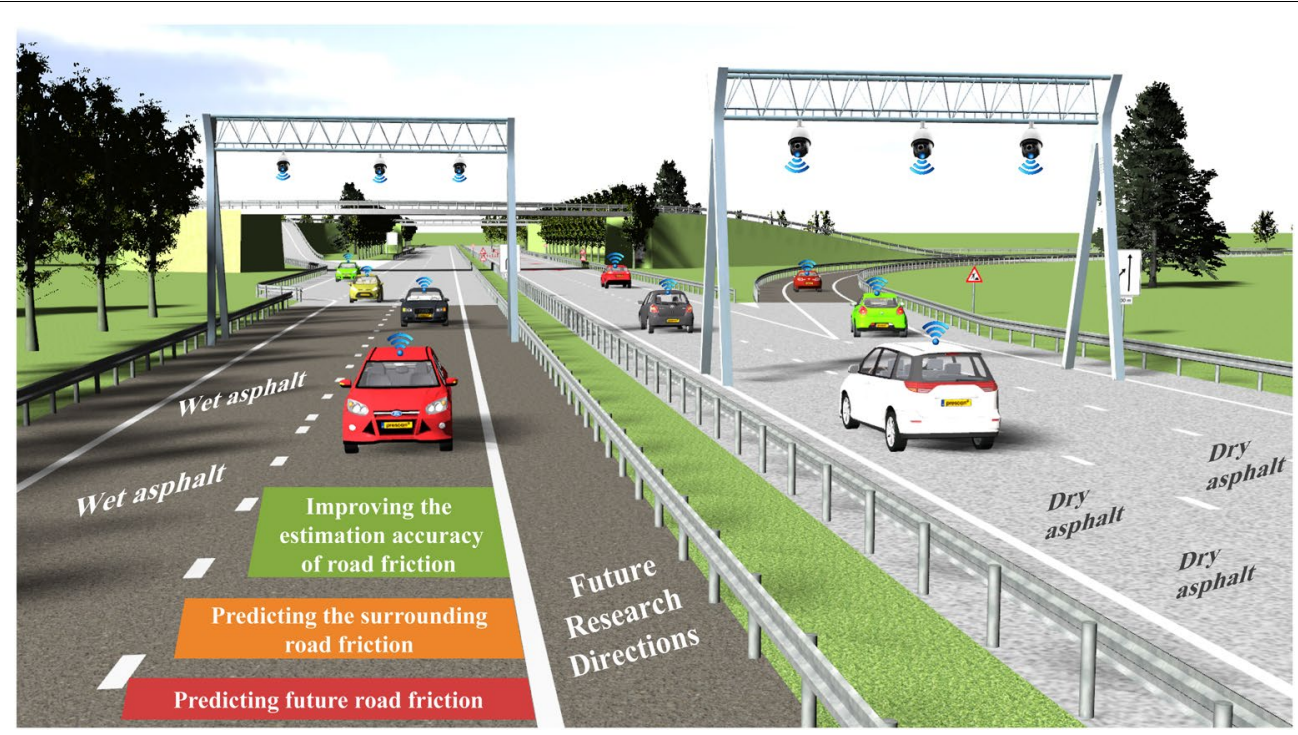

Figure 6 Future research directions on TRFC estimation 
(1) Off-board sensors-based estimation methods can obtain road friction information without any excitation, the disadvantage is that it is easily disturbed by the environment. Although the dynamics-based estimation method has strong robustness to environmental interference, it needs suitable excitation to obtain the ideal estimation performance. Furthermore, in the vehicle dynamics-based approaches, the simplification of the model often leads to a decrease in the accuracy of TRFC estimation. A data-driven approach can effectively solve the problem but its performance is heavily dependent on the integrity of the dataset. Therefore, it is necessary to combine different types of estimation methods with well-designed fusion rules to obtain good prediction performance. For example, the effective integration of vehicle dynamics-based approaches with sensing approaches to improve estimation accuracy is one of the popular research directions.

(2) With the development of mobile communication technology, vehicles can exchange data with various elements in the intelligent transportation system, including other vehicles, Internet gateways, and transport infrastructure. When some sensors on the vehicle fail, it can exchange information with other vehicles to obtain TRFC. In addition, the vehicle can also modify the estimated value of TRFC by exchanging data with the camera on the signal light. This means that the road friction of the adjacent road of the vehicle can also be obtained, which will help the driver to plan the driving route reasonably. Therefore, it is a promising research direction to integrate multiple information to predict the TRFC of local roads in a networked environment.

(3) Existing studies on TRFC estimation can only estimate the current road conditions based on the current sensor measurements and they cannot predict the future road conditions. Accurate prediction of TRFC in the next few days allows travelers and road managers to rationalize their trips and road maintenance activities, which contributes to the safety and efficiency of traffic. Combining historical road condition data with weather forecast data and using a data-driven method to estimate long-term TRFC is also an interesting research direction.

\section{Acknowledgments}

Not applicable.

\section{Authors' Contributions}

GY was in charge of the whole trial; YW wrote the manuscript; JH and FW assisted the trial; $H D, Y Y, Y R$, and $C Z$ conducted proofreading and made some critical revisions. All authors read and approved the final manuscript.

\section{Authors' Information}

Yan Wang, born in 1992, is currently a PhD candidate at the School of Mechanical Engineering, Southeast University, Nanjing, China. He received his M.S. degree in vehicle engineering from the Nanjing University of Aeronautics and Astronautics, China, in 2018. His research interests include vehicle state and parameter estimation, automotive active safety control. Tel: +86-13645165076. Jingyu Hu,born in 1997, is currently a master candidate at the School of Mechanical Engineering, Southeast University, Nanjing, China. He received his B.E. degree in mechanical engineering from Qingdao University, China, in 2019. His current research interests include intelligent driving,pose estimation algorithm and automotive active safety control. Tel: +86-13815564119.

Fa'an Wang, born in 1990, is currently a PhD candidate at the School of Mechanical Engineering, Southeast University, China. He received his M.S. degree in agricultural engineering from Kunming University of Science and Technology, China, in 2017. His current research interests include vehicle cooperative localization and control, intelligent connected vehicles. Tel: +86-18317710956; Haoxuan Dong, born in 1993, is currently a PhD candidate at the School of Mechanical Engineering, Southeast University, China. He received his M.S. degree in vehicle engineering from Chang'an University, China, in 2018. His research interests include connected vehicles, vehicle energy management, eco-driving control.Tel: +86-18363623668.

Yongjun Yan, born in 1995, is currently a PhD candidate at the School of Mechanical Engineering, Southeast University, China. He received his M.S. degree in vehicle engineering from Southeast University, China, in 2020. His current research interests include vehicle dynamics and control, automotive active safety control, and human-like driving. Tel: +86-15895991671.

Yanjun Ren, born in 1994, is currently a PhD candidate at the School of Mechanical Engineering, Southeast University, China. He received his M.S. degree in vehicle engineering from Southeast University, China, in 2020. His research interests include vehicle dynamics and control, automotive electrical and electronic system. Tel: +86-13913365373.

Chaobin Zhou, born in 1983, is currently a PhD candidate at the School of Mechanical Engineering, Southeast University, China. He received his M.S. degree in mechanical engineering from the Chang'an University, China, in 2010. His research interests include vehicle state and parameter estimation, automotive suspension system design. Tel: +86-18394029587.

Guodong Yin, born in 1976, is currently a Professor at the School of Mechanical Engineering, Southeast University, China. He received a PhD degree in vehicle engineering from Southeast University, China, in 2007. His current research interests include vehicle dynamics and control, connected vehicles, and multiagent control.Tel: +86-13913879060

\section{Funding}

Supported by the National Natural Science Funds for Distinguished Young

Scholar of China (Grant No. 52025121), National Natural Science Foundation of China (Grant Nos. 51975118, 52002066).

Availability of Data and Materials

The datasets supporting the conclusions of this article are included within the article.

\section{Competing Interests}

The authors declare no competing financial interests.

\section{Author Details \\ ${ }^{1}$ School of Mechanical Engineering, Southeast University, Nanjing 211189, China. ${ }^{2}$ College of Mechano-Electronic Engineering, Lanzhou University of Technology, Lanzhou, Gansu 730050, China.}

Received: 4 May 2021 Revised: 24 December 2021 Accepted: 30 December 2021

Published online: 31 January 2022

\section{References}

[1] A Patil, D Ginoya, P D Shendge, et al. Uncertainty-estimation-based approach to antilock braking systems. IEEE Transactions on Vehicular Technology, 2016, 65(3): 1171-1185. 
[2] W Zhao, X Qin, C Wang. Yaw and lateral stability control of automotive four-wheel steer-by-wire system. IEEE/ASME Transactions on Mechatronics, 2018, 23(6): 2628-2637.

[3] E Hashemi, M Jalali, A Khajepour, et al. Vehicle stability control: Model predictive approach and combined-slip effect. IEEE/ASME Transactions on Mechatronics, 2020, 25(6): 2789-2800.

[4] Y Wang, G Yin, Y Li, et al. Self-learning control for coordinated collision avoidance of automated vehicles. Proc IMechE Part D: J Automobile Engineering, 2021, 235(4): 1149-1163.

[5] L Li, F Wang, Q Zhou. Integrated longitudinal and lateral tire/road friction modeling and monitoring for vehicle motion control. IEEE Transactions on Intelligent Transportation Systems, 2006, 7(1): 1-19.

[6] M Acosta, S Kanarachos, M Blundell. Road friction virtual sensing: A review of estimation techniques with emphasis on low excitation approaches. Applied Sciences-Basel, 2017, 7(12): 1-47.

[7] S Khaleghian, A Emami, S Taheri. A technical survey on tire-road friction estimation. Friction, 2017, 5(2): 123-146.

[8] M Kanafi, A Kuosmanen, T Pellinen, et al. Macro- and micro-texture evolution of road pavements and correlation with friction. International Journal of Pavement Engineering, 2015, 16(2): 168-179.

[9] G Baffet, A Charara, G Dherbomez. An observer of tire-road forces and friction for active security vehicle systems. IEEE/ASME Transactions on Mechatronics, 2007, 12(6): 651-661.

[10] Y Du, C Liu, Y Song, et al. Rapid estimation of road friction for anti-skid autonomous driving. IEEE Transactions on Intelligent Transportation Systems, 2020, 21(6): 2461-2470.

[11] B Leng, D Jin, L Xiong, et al. Estimation of tire-road peak adhesion coefficient for intelligent electric vehicles based on camera and tire dynamics information fusion. Mechanical Systems and Signal Processing, 2021, 150: 107275.

[12] M Yu, XXu, CWu, et al. Research on the prediction model of the friction coefficient of asphalt pavement based on tire-pavement coupling. Advances in Materials Science and Engineering, 2021: 6650525.

[13] R Matsuzaki, K Kamai, R Seki. Intelligent tires for identifying coefficient of friction of tire/road contact surfaces using three-axis accelerometer. Smart Materials and Structures, 2015, 025010:1-9.

[14] K Singh, M Arat, S Taheri. An intelligent tire based tire-road friction estimation technique and adaptive wheel slip controller for antilock brake system. Journal of Dynamic Systems Measurement and Control-Transactions of the ASME, 2013, 135(3): 31002-31002.

[15] S Hong, G Erdogan, K Hedrick, et al. Tyre-road friction coefficient estimation based on tyre sensors and lateral tyre deflection: Modeling, simulations, and experiments. Vehicle System Dynamics, 2013, 51(5): 627-647.

[16] A Niskanen, A Tuononen. Three three-axis IEPE accelerometers on the inner liner of a tire for finding the tire-road friction potential indicators. Sensors, 2015, 15(8): 19251-19263.

[17] M Kim, J Park, S Choi. Road type identification ahead of the tire using D-CNN and reflected ultrasonic signals. International Journal of Automotive Technology, 2021, 22(1): 47-54.

[18] M Ergun, S Iyinam, A F lyinam. Prediction of road surface friction coefficient using only macro- and microtexture measurements. Journal of Transportation Engineering-ASCE, 2005, 131(4): 311-319.

[19] G Erdogan, L Alexander, R Rajamani. Estimation of tire-road friction coefficient using a novel wireless piezoelectric tire sensor. IEEE Sensors Journal, 2011, 11(2): 267-279.

[20] J H Yoon, S E Li, C Ahn. Estimation of vehicle sideslip angle and tireroad friction coefficient based on magnetometer with GPS. International Journal of Automotive Technology, 2016, 17(3): 427-435.

[21] F Gustafsson. Slip-based tire road friction estimation. Automatica, 1997, 33(6): 1087-1099.

[22] F Gustafsson. Monitoring tire-road friction using the wheel slip. IEEE Control Systems Magazine, 1998, 18(4): 42-49.

[23] KYi, T Jeong. Observer based estimation of tire-road friction for collision warning algorithm adaptation. JSME International Journal Series C-Mechanical Systems Machine Elements and Manufacturing, 1998, 41(1): $116-124$.

[24] KYi, K Hedrick, S C Lee. Estimation of tire-road friction using observer based identifiers. Vehicle System Dynamics, 1999, 31(4): 233-261.

[25] J M Wang, L Alexander, R Rajamani. Friction estimation on highway vehicles using longitudinal measurements. Journal of Dynamic Systems
Measurement and Control-Transactions of the ASME, 2004, 126(2): 265-275.

[26] R Rajamani, D Piyabongkarn, JY Lew, et al. Tire-road friction-coefficient estimation. IEEE Control Systems Magazine, 2010, 30(4): 54-69.

[27] D Paul, E Velenis, F Humbert, et al. Tyre-road friction MU-estimation based on braking force distribution. Proceedings of the Institution of Mechanical Engineers Part D-Journal of Automobile Engineering, 2019, 233(8): 2030-2047.

[28] C Lee, K Hedrick, K S Yi. Real-time slip-based estimation of maximum tire-road friction coefficient. IEEE/ASME Transactions on Mechatronics, 2004, 9(2): 454-458.

[29] K Li, J A Misener, K Hedrick. On-board road condition monitoring system using slip-based tyre-road friction estimation and wheel speed signal analysis. Proceedings of the Institution of the Institution of Mechanical Engineers Part K-Journal of Multi-Body Dynamics, 2007, 221(1): 129-146.

[30] R.D Castro, R.E Araujo, D Freitas. Real-time estimation of tyre-road friction peak with optimal linear parameterization. IET Control Theory and Applications, 2012, 6(14): 2257-2268.

[31] G J Cui, J L Dou, S S Li, et al. Slip control of electric vehicle based on tire-road friction coefficient estimation. Mathematical Problems in Engineering, 2017: 3035124.

[32] ]Y Hwang, S B Choi. Adaptive collision avoidance using road friction information. IEEE Transactions on Intelligent Transportation Systems, 2019 20(1): 348-361

[33] W.L Yong, X Guan, B Wang, et al. Research on the real-time identification approach of longitudinal road slope and maximum road friction coefficient. International Journal of Vehicle Design, 2019, 79(1): 18-42.

[34] G L Goodenow, TR Kolhoff, F D Smithson. Tire-road friction measuring system - a second generation. SAE Transactions, 1968, 77 . 680005-680246.

[35] M Sjahdanulirwan. An analytical model for the prediction of tyre road friction under braking and cornering. International Journal of Vehicle Design, 1993, 14(1): 78-99.

[36] M Sjahdanulirwan, Q J Yang. Prediction of tyre road friction with an inverted-boat shaped pressure distribution. Vehicle System Dynamics, 1995, 24(2): 145-161.

[37] C. S Liu, H Peng. Road friction coefficient estimation for vehicle path prediction. Vehicle System Dynamics, 1996, 25: 413-425.

[38] S Muller, M Uchanski, K Hedrick. Estimation of the maximum tire-road friction coefficient. Journal of Dynamic Systems Measurement and Control-Transactions of the ASME, 2003, 125(4): 607-617.

[39] G Erdogan, L Alexander, R Rajamani. Adaptive vibration cancellation for tire-road friction coefficient estimation on winter maintenance vehicles. IEEE Transactions on Control Systems Technology, 2010, 18(5): 1023-1032.

[40] J Pokorski, A Renski, H Sar. System for investigation of friction properties of the road surface. Baltic Journal of Road and Bridge Engineering, 2015, 10(2): 126-131.

[41] J G Yi, L Alvarez, C Claeys, et al. Emergency braking control with an observer-based dynamic tire/road friction model and wheel angular velocity measurement. Vehicle System Dynamics, 2003, 39(2): 81-97.

[42] L Alvarez, J G Yi, R Horowitz, et al. Dynamic friction model-based tireroad friction estimation and emergency braking control. Journal of Dynamic Systems Measurement and Control-Transactions of the Asme, 2005, 127(1): 22-32.

[43] N Patel, C Edwards, S.K Spurgeon. Tyre-road friction estimation - a comparative study. Proceedings of the Institution of Mechanical Engineers Part D-Journal of Automobile Engineering, 2008, 222(12): 2337-2351.

[44] G Baffet, A Charara, G Dherbomez. An observer of tire-road forces and friction for active security vehicle systems. IEEE/ASME Transactions on Mechatronics, 2007, 12(6): 651-661.

[45] N G Ding, G ZYu, W D Wang. Estimation of brake pressure and tyreroad friction during $A B S$ activation. International Journal of Vehicle Design, 2012, 58(1): 33-45.

[46] XW Zhang, Y Xu, M Pan, et al. A vehicle ABS adaptive sliding-mode control algorithm based on the vehicle velocity estimation and tyre/ road friction coefficient estimations. Vehicle System Dynamics, 2014, 52(4): 475-503

[47] XXia, L Xiong, K Sun, et al. Estimation of maximum road friction coefficient based on Lyapunov method. International Journal of Automotive Technology, 2016, 17(6): 991-1002. 
[48] B Y Li, H P Du, W H Li, et al. Comparative study of vehicle tyre-road friction coefficient estimation with a novel cost-effective method. Vehicle System Dynamics, 2014, 52(8): 1066-1098.

[49] Y Q Zhao, H Q Li, F Lin, et al. Estimation of road friction coefficient in different road conditions based on vehicle braking dynamics. Chinese Journal of Mechanical Engineering, 2017, 30(4): 982-990.

[50] J J Rath, K C Veluvolu, M Defoort. Simultaneous estimation of road profile and tire road friction for automotive vehicle. IEEE Transactions on Vehicular Technology, 2015, 64(10): 4461-4471.

[51] D J Lee, Y S Park. Sliding-mode-based parameter identification with application to tire pressure and tire-road friction. International Journal of Automotive Technology, 2011, 12(4): 571-577.

[52] M Tanelli, L Piroddi, S M Savaresi. Real-time identification of tire-road friction conditions. IET Control Theory and Applications, 2009, 3(7): 891-906.

[53] M Sharifzadeh, A Senatore, A Farnam, et al. A real-time approach to robust identification of tyre-road friction characteristics on mixedMU roads. Vehicle System Dynamics, 2019, 57(9): 1338-1362.

[54] R Rajamani, G Phanomchoeng, D Piyabongkarn, et al. Algorithms for real-time estimation of individual wheel tire-road friction coefficients. IEEE/ASME Transactions on Mechatronics, 2012, 17(6): 1183-1195.

[55] S Ko, J Ko, S Lee, et al. A study on the road friction coefficient estimation and motor torque control for an in-wheel electric vehicle. Proceedings of the Institution of Mechanical Engineers Part D-Journal of Automobile Engineering, 2015, 229(5): 611-623.

[56] K Han, Y Hwang, E Lee, et al. Robust estimation of maximum tire-road friction coefficient considering road surface irregularity. International Journal of Automotive Technology, 2016, 17(3): 415-425.

[57] J Zhao, J Zhang, B Zhu. Coordinative traction control of vehicles based on identification of the tyre-road friction coefficient. Proceedings of the Institution of Mechanical Engineers Part D-Journal of Automobile Engineering, 2016, 230(12): 1585-1604.

[58] K Han, E Lee, M Choi, et al. Adaptive scheme for the real-time estimation of tire-road friction coefficient and vehicle velocity. IEEE/ASME Transactions on Mechatronics, 2017, 22(4): 1508-1518.

[59] H Guan, B Wang, P P Lu, et al. Identification of maximum road friction coefficient and optimal slip ratio based on road type recognition. Chinese Journal of Mechanical Engineering, 2014, 27(5): 1018-1026.

[60] C S Kim, J O Hahn, K S Hong, et al. Estimation of tire-road friction based on on-board 6-DOF acceleration measurement. IEEE Transactions on Vehicular Technology, 2015, 64(8): 3368-3377.

[61] K Enisz, I Szalay, G Kohlrusz, et al. Tyre-road friction coefficient estimation based on the discrete-time extended Kalman filter. Proceedings of the Institution of Mechanical Engineers Part D-Journal of Automobile Engineering, 2015, 229(9): 1158-1168.

[62] J J Castillo, J A Cabrera, A J Guerra, et al. A novel electrohydraulic brake system with tire-road friction estimation and continuous brake pressure control. IEEE Transactions on Industrial Electronics, 2016, 63(3): 1863-1875.

[63] L R Ray. Nonlinear tire force estimation and road friction identification: Simulation and experiments. Automatica, 1997, 33(10): 1819-1833.

[64] LChen, M Y Bian, Y G Luo, et al. Tire-road friction coefficient estimation based on the resonance frequency of in-wheel motor drive system. Vehicle System Dynamics, 2016, 54(1): 1-19.

[65] A J C Schmeitz, M Alirezaei. Analysis of wheel speed vibrations for road friction classification. Vehicle System Dynamics, 2016, 54(4): 492-509.

[66] D Paul, E Velenis, D P Cao, et al. Optimal MU-estimation-based regenerative braking strategy for an AWD HEV. IEEE Transactions on Transportation Electrification, 2017, 3(1): 249-258.

[67] A Albinsson, F Bruzelius, B Jacobson, et al. Design of tyre force excitation for tyre-road friction estimation. Vehicle System Dynamics, 2017, 55(2): 208-230.

[68] S Yamazaki, O Furukawa, T Suzuki. Study on real time estimation of tire to road friction. Vehicle System Dynamics, 1997, 27: 225-233.

[69] X J Gao, Z P Yu, J Neubeck, et al. Sideslip angle estimation based on input-output linearization with tire-road friction adaptation. Vehicle System Dynamics, 2010, 48(2): 217-234.

[70] R R Wang, J M Wang. Tire-road friction coefficient and tire cornering stiffness estimation based on longitudinal tire force difference generation. Control Engineering Practice, 2013, 21(1): 65-75.
[71] KWakamatsu, Y Akuta, M Ikegaya, et al. Adaptive yaw rate feedback 4WS with tire/road friction coefficient estimator. Vehicle System Dynamics, 1997, 27(5-6): 305-326.

[72] O Nishihara, K Masahiko. Estimation of road friction coefficient based on the brush model. Journal of Dynamic Systems Measurement and Control-Transaction of the ASME, 2011, 33(4): 1-9.

[73] C E Beal. Rapid road friction estimation using independent left/right steering torque measurements. Vehicle System Dynamics, 2020, 58(3): 377-403.

[74] J O Hahn, R Rajamani, L Alexander. GPS-based real-time identification of tire-road friction coefficient. IEEE Transactions on Control Systems Technology, 2002, 10(3): 331-343.

[75] G Erdogan, L Alexander, R Rajamani. Friction coefficient measurement for autonomous winter road maintenance. Vehicle System Dynamics, 2009, 47(4): 497-512.

[76] L Li, H Z Li, J Song, et al. Road friction estimation under complicated maneuver conditions for active yaw control. Chinese Journal of Mechanical Engineering, 2009, 22(4): 514-520.

[77] L Li, J Song, $\mathrm{H}$ Z Li, et al. Comprehensive prediction method of road friction for vehicle dynamics control. Proceedings of the Institution of Mechanical Engineers Part D-Journal of Automobile Engineering, 2009, 223(D8): 987-1002

[78] L Li, G Jia, X Ran, et al. A variable structure extended Kalman filter for vehicle sideslip angle estimation on a low friction road. Vehicle System Dynamics, 2014, 52(2): 280-308.

[79] B Kwak, Y Park. Vehicle states observer using adaptive tire-road friction estimator. JSME International Journal Series C-Mechanical Systems Machine Elements and Manufacturing, 2001, 44(3): 668-675.

[80] N G Ding, STaheri. Application of recursive least square algorithm on estimation of vehicle sideslip angle and road friction. Mathematical Problems in Engineering, 2010: 242-256.

[81] C F Zong, P Song, D Hu. Estimation of vehicle states and tire-road friction using parallel extended Kalman filtering. Journal of Zhejiang University-Science A, 2011, 12(6): 446-452.

[82] Y Xu, B.Y Chen, C Chi. Estimation of road friction coefficient and vehicle states by 3-DOF dynamic model and HSRI model based on information fusion. Asian Journal of Control, 2018, 20(3): 1067-1076.

[83] J Q Hu, S Rakheja, Y M Zhang. Real-time estimation of tire-road friction coefficient based on lateral vehicle dynamics. Proceedings of the Institution of Mechanical Engineers Part D-Journal of Automobile Engineering, 2020, 234(10-11): 2444-2457.

[84] Y H Liu, T Li, Y Y Yang, et al. Estimation of tire-road friction coefficient based on combined APF-IEKF and iteration algorithm. Mechanical Systems and Signal Processing, 2017, 88: 25-35.

[85] N G Ding, W Chen, Y P Zhang, et al. An extended Luenberger observer for estimation of vehicle sideslip angle and road friction. International Journal of Vehicle Design, 2014, 66(4): 385-414.

[86] WW Chen, D KTan, L F Zhao. Vehicle sideslip angle and road friction estimation using online gradient descent algorithm. IEEE Transactions on Vehicular Technology, 2018, 67(12): 11475-11485.

[87] L T Gao, L Xiong, X F Lin, et al. Multi-sensor fusion road friction coefficient estimation during steering with Lyapunov method. Sensors, 2019, 19(18): 1-17.

[88] L Shao, C Jin, A Eichberger, et al. Grid search based tire-road friction estimation. IEEE Access, 2020, 8: 81506-81525.

[89] L Etienne, C A Lua, S Di Gennaro, et al. A super-twisting controller for active control of ground vehicles with lateral tire-road friction estimation and CarSim validation. International Journal of Control Automation and Systems, 2020, 18(5): 1177-1189.

[90] S Solmaz, S C Baslamisli. Simultaneous estimation of road friction and sideslip angle based on switched multiple non-linear observers. IET Control Theory and Applications, 2012, 6(14): 2235-2247.

[91] L Shao, C Jin, C Lex, et al. A robust road friction estimation during vehicle steering. Vehicle System Dynamics, 2019, 57(4): 493-519.

[92] M Choi, J J Oh, S B Choi. Linearized recursive least squares methods for real-time identification of tire-road friction coefficient. IEEE Transactions on Vehicular Technology, 2013, 62(7): 2906-2918.

[93] T Shim, D Margolis. Model-based road friction estimation. Vehicle System Dynamics, 2004, 41(4): 249-276. 
[94] J Villagra, B Andrea-Novel, M Fliess, et al. A diagnosis-based approach for tire-road forces and maximum friction estimation. Control Engineering Practice, 2011, 19(2): 174-184.

[95] C Ahn, H Peng, H ETseng. Robust estimation of road friction coefficient using lateral and longitudinal vehicle dynamics. Vehicle System Dynamics, 2012, 50(6): 961-985.

[96] H B Ren, S Z Chen, T Shim, et al. Effective assessment of tyre-road friction coefficient using a hybrid estimator. Vehicle System Dynamics, 2014, 52(8): 1047-1065.

[97] Y C Feng, H Chen, HY Zhao, et al. Road tire friction coefficient estimation for four-wheel drive electric vehicle based on moving optimal estimation strategy. Mechanical Systems and Signal Processing, 2020, 139:1-23.

[98] L Li, K Yang, G Jia, et al. Comprehensive tire-road friction coefficient estimation based on signal fusion method under complex maneuvering operations. Mechanical Systems and Signal Processing, 2015, 56-57: 259-276.

[99] L Chen, M.Y Bian, Y G Luo, et al. Real-time identification of the tyre-road friction coefficient using an unscented Kalman filter and mean-squareerror-weighted fusion. Proceedings of the Institution of Mechanical Engineers Part D-Journal of Automobile Engineering, 2016, 230(6): 788-802.

[100] L Chen, Y G Luo, M Y Bian, et al. Estimation of tire-road friction coefficient based on frequency domain data fusion. Mechanical Systems and Signal Processing, 2017, (85): 177-192.

[101] J Zhao, J Zhang, B Zhu. Development and verification of the tire/road friction estimation algorithm for antilock braking system. Mathematical Problems in Engineering, 2014: 786492.

[102] A K Madhusudhanan, M Corno, M A Arat, et al. Load sensing bearing based road-tyre friction estimation considering combined tyre slip. Mechatronics, 2016, (39): 136-146.

[103] C F Zong, D Hu, HY Zheng. Dual extended Kalman filter for combined estimation of vehicle state and road friction. Chinese Journal of Mechanical Engineering, 2013, 26(2): 313-324.

[104] Z.Q Qi, S Taheri, B.F Wang, et al. Estimation of the tyre-road maximum friction coefficient and slip slope based on a novel tyre model. Vehicle System Dynamics, 2015, 53(4): 506-525.

[105] B Huang, X Fu, SWu, et al. Calculation algorithm of tire-road friction coefficient based on limited-memory adaptive extended Kalman filter. Mathematical Problems in Engineering, 2019: 1056269.

[106] ]T Nakatsuji, I Hayashi, P Ranjitkar, et al. Online estimation of friction coefficients of winter road surfaces using the unscented Kalman filter. Transportation Research Record, 2007, 2015: 113-122.

[107] XY Ping, S Cheng, W Yue, et al. Adaptive estimations of tyre-road friction coefficient and body's sideslip angle based on strong tracking and interactive multiple model theories. Proceedings of the Institution of Mechanical Engineers Part D-Journal of Automobile Engineering, 2020: $1-15$.

[108] C F Chen, Y M Jia, Y L Wang, et al. Non-linear velocity observer for vehicles with tyre-road friction estimation. International Journal of Systems Science, 2018, 49(7): 1403-1418.

[109] Y Peng, J Chen, Y Ma. Observer-based estimation of velocity and tireroad friction coefficient for vehicle control systems. Nonlinear Dynamics, 2019, 96(1): 363-387.

[110] A Zareian, S Azadi, R Kazemi. Estimation of road friction coefficient using extended Kalman filter, recursive least square, and neural network. Proceedings of the Institution of Mechanical Engineers Part K-Journal of Multi-Body Dynamics, 2016, 230(1): 52-68.

[111] W R Pasterkamp, H B Pacejka. Optimal design of neural networks for estimation of tyre/road friction. Vehicle System Dynamics, 1998, 29: 312-321.

[112] N Takashi, H Ikuko, K Akira, et al. Inverse estimation of friction coefficients of winter road surfaces - New considerations of lateral movements and angular movements. Maintenance Management and Operations Services, 2005, 1911(1): 149-159.

[113] X D Zhang, D Gohlich. A hierarchical estimator development for estimation of tire-road friction coefficient. Plos One, 2017, 12(2): 1-21.

[114] A M Ribeiro, A Moutinho, A R Fioravanti, et al. Estimation of tire-road friction for road vehicles: A time delay neural network approach. Journal of the Brazilian Society of Mechanical Sciences and Engineering, 2020, 42(4):1-12.
[115] ZY Pu, ZY Cui, SWang, et al. Time-aware gated recurrent unit networks for forecasting road surface friction using historical data with missing values. IET Intelligent Transport Systems, 2020, 14(4): 213-219.

[116] E Sabanovic, V Zuraulis, O Prentkovskis, et al. Identification of roadsurface type using deep neural networks for friction coefficient estimation. Sensors, 2020, 20(3): 1-17.

[117] ZY Pu, C L Liu, X M Shi, et al. Road surface friction prediction using long short-term memory neural network based on historical data. Journal of Intelligent Transportation Systems, 2020: 1-12.

\section{Submit your manuscript to a SpringerOpen ${ }^{\circ}$ journal and benefit from:}

- Convenient online submission

- Rigorous peer review

- Open access: articles freely available online

- High visibility within the field

- Retaining the copyright to your article

Submit your next manuscript at $\boldsymbol{\nabla}$ springeropen.com 